\title{
Zur Revisionspraxis der VGR der Länder
}

\author{
Roland Döhrn
}

Eingegangen: 2. November 2020 / Angenommen: 13. März 2021 / Online publiziert: 9. April 2021

(C) Der/die Autor(en) 2021

Zusammenfassung Die Volkswirtschaftlichen Gesamtrechnungen der Länder werden oft als Erfolgsnachweis der Wirtschaftspolitik eines Bundeslandes herangezogen. Allerdings werden die Angaben im Laufe der Zeit erheblich revidiert, wodurch sich die Positionierung der Länder in einer „Wachstumsrangliste“ beträchtlich ändern kann. Der vorliegende Beitrag analysiert Revisionen der VGR der Länder im Zeitraum 2000 bis 2017. Er zeigt, dass die Angaben zum BIP kräftiger revidiert werden als die zum Arbeitsmarkt, so dass die daraus abgeleitete Produktivität im Zuge des Revisionsprozesses stark variiert. Besonders ausgeprägt sind die Revisionen aller Größen beim Baugewerbe. Alles in allem liefert die Evaluation der Revisionspraxis Hinweise, wie die Revisionen der VGR der Länder verringert werden könnten. So sollten den Angaben zum Arbeitsmarkt ein höheres Gewicht beigemessen, da sie weniger revisionsanfällig sind und besser einzelnen Regionen zugeordnet werden können. Zudem sollte die Produktivität als Kontrollgröße verwendet werden.

Schlüsselwörter VGR der Länder · Datenrevisionen

JEL-Klassifikation C82 $\cdot$ E01 · E66

R. Döhrn (凹)

RWI Leibniz-Institut für Wirtschaftsforschung, Essen, Deutschland

E-Mail: doehrn@rwi-essen.de 


\title{
Revision practice of the National Accounts of the German federal states
}

\begin{abstract}
The national accounts of the federal states are often used as proof of the success of the economic policy of a federal state. However, the data will be revised considerably after the first publication. As a result, the positioning of the countries in a "growth ranking" changes significantly over time. This article analyzes revisions of the national accounts of the federal states, in the period 2000 to 2017. It shows that the data on GDP are revised more strongly than those on the labor market, so that the productivity derived from them varies greatly in the course of the revision process. All revisions are particularly pronounced in the construction sector. All in all, the evaluation of the revision practice provides information on how the revisions of the national accounts of the federal states could be reduced. For example, more weight should be attached to the labor market information that is less prone to revision and can be allocated better to regions. Furthermore, productivity should be used as control parameter.
\end{abstract}

Keywords National accounts of federal states $\cdot$ Data revisions

\section{Einleitung}

Die Ergebnisse der Volkswirtschaftlichen Gesamtrechnungen (VGR) der Länder spielen in der politischen Diskussion eine nicht zu unterschätzende Rolle. Sie sind ein zentraler Maßstab der Wirtschaftskraft von Bundesländern und Regionen, und gehen deshalb beispielsweise in diverse Rankings ein. ${ }^{1}$ Selten problematisiert wird die Aussagekraft dieser Daten, und dies, obwohl sie auf dem Weg von der ersten Veröffentlichung bis zur endgültigen Zahl substanziellen Revisionen unterworfen sind.

So wurde im März 2016 für Nordrhein-Westfalen eine Zuwachsrate des realen Bruttoinlandsprodukts (BIP) im Jahr 2015 von Null ausgewiesen, bei einer Zunahme des deutschen BIP um 1,7\%. Dies löste in NRW eine heftige „Nullwachstumsdebatte“ aus. Heute wird eine Zunahme um $1 \%$ ausgewiesen, bei unveränderter BIP-Rate für Deutschland. Damit liegt die Expansion in NRW zwar immer noch unter dem Bundesdurchschnitt, der Abstand erscheint aber weniger besorgniserregend. Umgekehrt wurde Zunahme des nordrhein-westfälischen BIP im Jahr 2016 von 1,8\% (veröffentlicht im März 2017) auf 1,1\% (Datenstand März 2020) nach unten revidiert. Im Gegenzug mutierte Niedersachsen, für das ursprünglich eine unterdurchschnittliche Zuwachsrate im Jahr 2016 von 1,4\% gemeldet worden war, mit einem (im März 2020 ausgewiesenen) Plus von 6,0\% zum Spitzenreiter in der Rangfolge der Bundesländer. ${ }^{2}$ Dabei ist zu beachten, dass bevölkerungsreiche Länder wie NRW

\footnotetext{
1 Ein Beispiel ist das Bundesländerranking der Initiative Neue Soziale Marktwirtschaft INSM (http:// bundeslaenderranking.de/).

2 Nach einer Pressemitteilung des Landesamtes für Statistik Niedersachsen ist diese Aufwärtsrevision auf einen deutlichen, und in die erste Berechnung nicht eingestellten Rückgang der Vorleistungsbezüge von Volkswagen zurückzuführen. (LSN 2018).
} 
und Niedersachsen bezüglich ihrer Wirtschaftsstruktur breit aufgestellt sind, was das Ausmaß späterer Revisionen begrenzt. In kleineren Ländern und Stadtstaaten konzentriert sich die Produktion oft auf wenige Branchen, was die Daten anfälliger für Revisionen macht.

Diese Beobachtungen legen eine systematische Auseinandersetzung mit den Revisionen der VGR der Länder nahe. Diese gibt es meines Wissens bisher nicht. In den Qualitätsberichten zu den VGR der Länder findet man zwar einige Angaben zum Ausmaß der Revisionen (AK VGRdL 2020, S. 16). Diese beziehen sich allerdings allein auf das BIP und lediglich auf Durchschnitte über alle Bundesländer; Aussagen zu einzelnen Ländern findet man nicht. Auch ist mit Blick die Aussagekraft der Daten von Interesse, die entstehungsseitigen Komponenten näher zu betrachten, etwa die Bruttowertschöpfung der Wirtschaftsbereiche oder Angaben zur Beschäftigung und zur Produktivität.

Bevor auf die Revisionen im Detail eingegangen wird, ist es zunächst angebracht, kurz, und soweit es für einen Ausstehenden möglich ist, auf den Rechengang bei der Erstellung der VGR der Länder einzugehen (Abschn. 2). Dabei wird auch auf mögliche Fehlerquellen und Revisionsursachen eingegangen. Im anschließenden Abschn. 3 werden die Revisionen des BIP und seiner Komponenten dargestellt. Den Ausgangspunkt bilden die nominalen Werte, da diese auch den Ausgangspunkt der Berechnungen des Arbeitskreises VGR bilden. Zudem werden Revisionen der Deflatoren, der Beschäftigung, des Arbeitsvolumens und der Produktivität analysiert. In Abschn. 4 wird dann auf drei Wirtschaftsbereiche, nämlich das Verarbeitende Gewerbe, das Baugewerbe und die Gesamtheit der Dienstleistungsbereiche eingegangen. Abgeschlossen wird das Papier mit Überlegungen, wie man Aussagekraft und Revisionsanfälligkeit der VGR der Länder verbessern könnte (Abschn. 5).

\section{Daten}

\subsection{Zur Erstellung der VGR der Länder}

Den folgenden Ausführungen ist voranzuschicken, dass der Autor nicht an der Erstellung der VGR der Länder beteiligt ist, und insofern den Rechenprozess nur anhand der veröffentlichten Unterlagen nachvollziehen kann, insbesondere der Methodenbeschreibung (AK VGRdL 2017) und der Qualitätsberichte (AK VGRdL 2020) des Arbeitskreises.. Er hat damit keinen Einblick in zusätzliche Abstimmungsprozesse und Kontrollrechnungen, die der Arbeitskreis VGR der Länder vornimmt, um seine Berechnungen zu plausibilisieren.

Die Erstellung der VGR der Länder erfolgt seit 2008 in fünf Rechenrunden. Die ersten Angaben zur Wirtschaftsleistung der deutschen Bundesländer werden in der Regel - bei Generalrevisionen der VGR kann der Termin abweichen - Ende März des dem jeweiligen Berichtsjahr folgenden Jahres veröffentlicht. ${ }^{3}$ Diese Daten basieren auf unvollständigen Daten, weshalb sie als erste Fortschreibung bezeichnet

\footnotetext{
3 Bis 2008 wurde die erste Fortschreibung bereits im Februar veröffentlicht. Ihr folgte bereits im März eine zweite Fortschreibung und im März des Folgejahres eine dritte.
} 
werden. Ein Jahr später, mit Veröffentlichung der ersten Fortschreibung für das dann neue Jahr, werden die Berechnungen für das Jahr davor revidiert. Da aber auch zu diesem Zeitpunkt noch wesentliche Basisstatistiken fehlen, wird diese zweite Veröffentlichung als zweite Fortschreibung bezeichnet. Erst ein Jahr später, also etwa 9 Quartale nach Abschluss des Berichtsjahres, liegen alle wesentlichen Basisstatistiken vor, so dass dann eine erste sog. Originärberechnung veröffentlicht werden kann.

Aber diese liefert noch keine endgültigen Werte. Dieser ersten folgen eine zweite und eine dritte Originärberechnung, mit denen zum Teil Revisionen der VGR für Deutschland insgesamt nachvollzogen werden, zum Teil weitere länderspezifische Informationen eingearbeitet werden. Im Zuge dieser Revisionen kommt es nochmals zu beträchtlichen Verschiebungen zwischen den Ländern. Das endgültige Ergebnis, also die dritte Originärberechnung, liegt erst im März des fünften Jahres nach Ende des Berichtsjahres fest. Damit ist der Revisionszyklus der VGR der Länder länger als der der gesamtdeutschen VGR, der im August des vierten Jahres nach Ende des Berichtsjahres abgeschlossen ist. Dies liegt daran, dass die VGR der Länder jeweils nur im März revidiert wird (im August jeden Jahres werden lediglich Daten zur Verwendungs- und Einkommensseite veröffentlicht), so dass die letzte Revision auf gesamtdeutscher Ebene erst ein gutes halbes Jahr später auf der Ebene der Länder nachvollzogen wird.

Die beschriebene Revisionsrunden beziehen sich auf die Entstehungsseite des BIP, die im Mittelpunkt der VGR der Länder steht, weil eine vollständige Verwendungsrechnung auf Länderebene aufgrund fehlender Angaben zu innerdeutschen „Außenhandels“-Strömen nicht kurzfristig zu erstellen ist. Die inländischen Verwendungskategorien werden erst mit zeitlichem Abstand veröffentlicht. Auf sie wird hier nicht eingegangen.

Die Entstehungsrechnung erfolgt auf der Ebene von Wirtschaftsbereichen, deren Wertschöpfung zur Bruttowertschöpfung des Landes zusammengefasst wird. Damit folgt die VGR der Länder im Prinzip einem bottom-up-Ansatz. Fehlen regionalspezifischen Daten, werden gesamtdeutsche Daten anhand geeigneter Schlüsselgrößen auf Regionen verteilt, weshalb das Vorgehen vom Arbeitskreis auch als Pseudobottom-up Ansatz bezeichnet wird (AK VGRdL 2017). Um zum BIP zu gelangen, werden zu der aggregierten Bruttowertschöpfung die Gütersteuern (abzüglich Gütersubventionen) hinzugezählt. ${ }^{4}$

Den Ausgangspunkt der Berechnungen bilden generell die Produktionswerte der im jeweiligen Land ansässigen Betriebe. Diese werden im Rahmen der Kostenstrukturerhebungen ermittelt, die erst etwa 6 Quartale nach Abschluss des Jahres vorliegen. Für die Fortschreibungen werden daher die Produktionswerte auf Basis der Umsatzentwicklung geschätzt. ${ }^{5}$ Dazu müssen zum einen Mehrbetriebsunternehmen auf die Länder aufgeschlüsselt werden. Zum anderen sind Hinzuschätzungen für im

\footnotetext{
${ }^{4}$ Dabei rechnet nicht jedes Bundesland für sich, sondern die einzelnen statistischen Ämter sind jeweils für einen oder mehrere Wirtschaftszweige zuständig und berechnen dessen Wertschöpfung für alle Länder.

5 Für die zweite Fortschreibung werden für das Verarbeitende Gewerbe außerdem auf Grundlage der zu diesem Zeitpunkt bereits vorliegenden Unternehmensangaben vorläufige Werte der Kostenstrukturerhebung (sog. Schnell-KSE) ermittelt (AK VGRdL 2017, Kap. 1, S. 4).
} 
Berichtskreis der jeweiligen Erhebungen fehlende Betriebe erforderlich. Speziell im Verarbeitenden Gewerbe erfolgt dabei die regionale Aufteilung anhand der Angaben zu den Löhnen und Gehältern. Die Produktionswerte werden um Vorleistungen vermindert, um zur Wertschöpfung zu gelangen. Da die Vorleistungsquoten ebenfalls erst im Rahmen der Kostenstrukturerhebungen anfallen, liegen erst für die erste Originärberechnung jahresspezifische Angaben vor. In den beiden Fortschreibungen werden historische Vorleistungsquoten verwendet.

Bei den Dienstleistungssektoren spielen zwar auch die Umsatzerhebungen im Dienstleistungsbereich und die Umsatzsteuerstatistik eine wichtige Rolle, jedoch werden hier in größerem Umfang Arbeitsmarktstatistiken und Verdiensterhebungen einbezogen, auf deren Grundlage die arbeitsbezogene Wertschöpfungskomponente abgeleitet werden kann. Umsatzsteuerstatistiken stoßen dort an Grenzen, wo manche Erbringer von Dienstleistungen nicht umsatzsteuerpflichtig sind, wie z.B. viele öffentliche Dienstleister und Gesundheitsdienstleister. Bei Letzteren werden auch die Abrechnungsstatistiken der kassenärztlichen Bundesvereinigung als Datenquelle genutzt. Teile der Wertschöpfung entstammen modellhaften Berechnungen, so der Mietwert der selbstgenutzten Wohnung im Bereich der Wohnungsvermietung und die unterstellten Bankgebühren (FISIM) für den Bereich der Kreditinstitute und Versicherungen.

Mit Blick auf den Revisionsbedarf und die Aussagekraft der VGR der Länder muss man zwei Probleme unterscheiden. Das erste resultiert daraus, dass viele Datenquellen - so die erwähnte Kostenstrukturerhebung - erst mit zeitlichem Abstand zum Berichtsjahr anfallen. Sie werden im Laufe der Zeit eingearbeitet und sind deshalb die wesentliche Ursache für Unterschiede zwischen der ersten und der zweiten Fortschreibung sowie der Originärberechnung. Das zweite Problem ist die Aufschlüsselung von Daten auf die Länderebene. Während arbeits-bezogene Wertschöpfungskomponenten sich über die Beschäftigungsstatistiken recht trennscharf zuordnen lassen, weil die sozialversicherungspflichtige Beschäftigung differenziert nach Arbeitsort vorliegt, gibt es bei kapital-bezogenen Wertschöpfungskomponenten ein Zurechnungsproblem, das auch mit einem Mehr an Daten schwer zu lösen ist.

Alle bisher beschriebenen Berechnungen legen jeweilige Preise zugrunde, beziehen sich also auf nominale Werte. Die Deflationierung, und damit der Übergang zu den in der Öffentlichkeit stärker beachteten preisbereinigten Werten, erfolgt in einem separaten Rechenschritt. Da auch die darin verwendeten Deflatoren revidiert werden, ergeben sich Revision z. B. des preisbereinigten BIP sowohl aus Revisionen der Nominalwerte als auch aus Revisionen der Deflatoren. Da keine länderspezifischen Preisinformationen vorliegen, lehnt sich der Arbeitskreis VGR der Länder an die entsprechenden Indizes auf Bundesebene an. Dabei verwendet er die jeweils tiefste Rechenebene der Bundesstatistik, d.h. Änderungen in der branchenmäßigen Zusammensetzung der Wirtschaftsleistung im Zuge der Revisionen schlagen sich auch in Änderungen des Deflators auf übergeordneter Ebene nieder, sofern sich die relativen Preise der Branchen unterschiedlich entwickeln.

In den VGR der Länder werden auch Angaben zur Erwerbstätigkeit und zum Arbeitsvolumen ausgewiesen. Diese werden allerdings nicht vom Arbeitskreis VGR der Länder erstellt, sondern vom Arbeitskreis Erwerbstätigenrechnung der Länder. Zwar gehen - wie erwähnt - in die Berechnung der Bruttowertschöpfung einiger Sektoren 
auch Informationen zum Arbeitsmarkt ein. Beide Arbeitskreise stützen sich auf unterschiedliche Ausgangsdaten, weshalb Änderungen in der Erwerbstätigenrechnung nicht notwendiger Weise auf die Gesamtrechnung durchschlagen, was sich auch für Deutschland insgesamt beobachten lässt (Döhrn 2019a, S. 110). Deshalb schlagen sich Änderungen in dem einen oder in dem anderen Rechenwerk jeweils in Änderungen der Produktivität nieder, ein Umstand, der unten zur Plausibilisierung der Daten genutzt wird.

\subsection{Methodische Überlegungen zur Revisionsanalyse}

Grundsätzlich muss man zwei Arten von Revisionen der VGR unterscheiden: Erstens die laufenden Revisionen, in denen, wie im vorhergehenden Abschnitt beschrieben, neue Daten in das Rechenwerk eingearbeitet werden. Zweitens finden im Abstand von durchschnittlich fünf Jahren sog. Generalrevisionen statt, in denen die Methodik der VGR grundlegend überarbeitet wird und neue Datenquellen einbezogen werden (Statistisches Bundesamt 2016, S. 30). Beide Revisionen überlagern sich, sollten aber, soll eine Revisionsanalyse Ansätze für eine Weiterentwicklung der VGR liefern, unterschieden werden. Denn nur die laufenden Revisionen lassen sich durch Verbesserungen der Datenerhebung oder der Datenauswertung verringern, während die Generalrevisionen sich überwiegend an konzeptionellen Überlegungen orientieren. Bei den VGR für Deutschland insgesamt kann man aufgrund der vierteljährlichen Erscheinungsweise beide Revisionen mit Hilfe einfacher Absätze näherungsweise trennen (Döhrn 2019a). Für die VGR der Länder können hingegen unterjährige Daten nicht für eine solche Unterscheidung herangezogen werden. ${ }^{6}$ Im Qualitätsbericht wird zwar der durchschnittliche Einfluss vergangener Generalrevisionen auf die BIP-Raten ausgewiesen (AK VGRdL 2020, S. 16). Die Zusammenstellung zeigt, dass dieser beträchtlich ist. Die Angaben beziehen sich aber zum einen auf einen Mittelwert über alle Bundesländer und geben zum anderen jeweils den Einfluss auf alle zurückliegenden Jahre an, weshalb sich die dort gemachten Angaben nicht auf einen konkreten Revisionszyklus übertragen lassen. Im Folgenden wird daher nicht zwischen laufenden Revisionen und Generalrevisionen unterschieden.

Zudem beschränken sich die folgenden Ausführungen auf einen Vergleich der Veränderungsraten gegenüber dem Vorjahr. Die sich im Laufe der Zeit ergebenden Änderungen der Niveaus der Realwerte aufgrund von Änderungen der Preisbasis, aber auch der Nominalwerte im Zuge definitorischer Änderungen im Rahmen der Generalrevisionen lassen eine Analyse der Revisionen der Niveaus nicht sinnvoll erscheinen.

\subsection{Verwendete Datenbasis}

Datengrundlage der folgenden Berechnungen sind die Veröffentlichungen des Arbeitskreises Volkswirtschaftliche Gesamtrechnungen der Länder. Zur Verfügung stehen Angaben für die Jahre 2000 bis 2019. Für Erwerbstätige, Arbeitsvolumen und

\footnotetext{
6 Zur Jahresmitte werden zwar BIP-Zahlen für die erste Hälfte des jeweils laufenden Jahres veröffentlicht. Diese werden aber später nicht mehr auf die sich ändernden Jahreswerte abgestimmt.
} 
folglich auch die Produktivität liegen allerdings für die frühen Jahres dieses Jahrtausends keine Angaben vor, so dass der Untersuchungszeitraum teilweise später beginnt. Da 2017 das letzte Jahr ist, für das die erste Originärberechnung vorliegt, endet der Untersuchungszeitraum in diesem Jahr. Dort, wo mit der dritten Originärberechnung verglichen wird, endet der Zeitraum bereits 2015.

Analysiert werden nicht nur das BIP insgesamt, sondern auch Angaben für die drei Wirtschaftsbereiche Verarbeitendes Gewerbe, Baugewerbe und Dienstleistungen. Für die Jahre vor 2006 enthalten die VGR der Länder allerdings keine Angaben zum Arbeitsmarktvariablen für das Verarbeitende Gewerbe. Hier ist der Untersuchungszeitraum daher teilweise kürzer.

Die Daten wurden soweit möglich den jeweils im März veröffentlichten Tabellen entnommen, die auf dem Rechenstand der gesamtdeutschen VGR vom August des Vorjahres bzw. von Februar des gleichen Jahres aufbauen (bis 2008 zweite Fortschreibung, danach erste Fortschreibung). Davon abgewichen werden musste in den Jahren, in denen im Zusammenhang mit der Umsetzung von Generalrevisionen die Daten verzögert oder nur unvollständig veröffentlicht wurden. Dadurch ergaben sich folgende Abweichungen:

- Im März 2006 wurden aufgrund der Generalrevision 2005 zunächst keine Angaben für das zurückliegende Jahr 2005 veröffentlicht. Hier wurden im August 2006 veröffentlichte Werte verwendet.

- Nach der Generalrevision 2011 publizierte der Arbeitskreis VGR der Länder im März 2012 nur Angaben für die Jahre 2008 bis 2011. Damit liegen keine Daten für die revidierten Veränderungsraten der Jahre 2007 und 2008 vor. Hier wurden die zu Jahresbeginn 2013 veröffentlichten Werte verwendet.

- Im Jahr 2017 wurde die Erwerbstätigenrechnung revidiert, weshalb in den die VGR der Länder vom März 2017 Angaben zu Beschäftigung und Produktivität im Jahr 2016 fehlen. Angaben zu den Erwerbstätigten veröffentlichte der Arbeitskreis Erwerbstätigenrechnung der Länder im Mai 2016. Daten zum Arbeitsvolumen fehlen; um keine Datenlücken entstehen zu lassen, wurden die im März 2018 veröffentlichten Angaben für das Jahr 2016 auch für den Veröffentlichungsjahrgang 2017 verwendet.

Überall dort, wo Bruttowertschöpfung und Beschäftigungsdaten aus unterschiedlichen Veröffentlichungen stammen, wurde die Produktivität errechnet, um eine konsistente Darstellung sicherzustellen.

\section{Revisionen des BIP auf Länderebene}

\subsection{Verzerrung und Ausmaß der Revisionen}

Bezugspunkt der folgenden Analysen ist jeweils die erste Originärberechnung. Zu ihr werden sowohl die beiden Fortschreibungen als auch die beiden anschließenden Revisionen in Relation gesetzt. Außerdem wird die sich insgesamt ergebende Revision betrachtet, also die Erste Fortschreibung mit der dritten Originärberechnung verglichen. Betrachtet werden vier Revisionsmaße: 
Tab. 1 Revisionen der Jahresraten des nominalen BIP der Länder. (Quelle: Eigene Berechnungen nach Angaben des Arbeitskreises VGR der Länder)

\begin{tabular}{|c|c|c|c|c|c|c|c|c|}
\hline & \multicolumn{4}{|c|}{$\begin{array}{l}\text { Erste Fortschreibung vs. erste Originär- } \\
\text { berechnung } \\
\text { 2000-2017 }\end{array}$} & \multicolumn{4}{|c|}{$\begin{array}{l}\text { Zweite Fortschreibung vs. erste Origi- } \\
\text { närberechnung } \\
\text { 2000-2017 }\end{array}$} \\
\hline & MR & MAR & MQR & NSR & MR & MAR & MQR & NSR \\
\hline $\begin{array}{l}\text { Baden- } \\
\text { Württem- } \\
\text { berg }\end{array}$ & $-0,06$ & 0,97 & 1,31 & 0,13 & $-0,19$ & 0,75 & 0,86 & 0,08 \\
\hline Bayern & 0,48 & 0,74 & 1,15 & 0,35 & 0,28 & 0,68 & 0,72 & 0,22 \\
\hline Berlin & 0,19 & 0,91 & 1,13 & 0,27 & 0,40 & 0,82 & 1,00 & 0,24 \\
\hline Brandenburg & 0,25 & 1,26 & 2,23 & 0,74 & 0,26 & 1,15 & 1,95 & 0,65 \\
\hline Bremen & $-0,53$ & 1,45 & 4,45 & 0,42 & $-0,37$ & 1,02 & 3,73 & 0,35 \\
\hline Hamburg & $-0,60$ & 1,29 & 2,05 & 0,43 & $-0,50$ & 1,33 & 2,26 & 0,47 \\
\hline Hessen & $-0,14$ & 0,90 & 1,05 & 0,30 & $-0,12$ & 0,58 & 0,61 & 0,17 \\
\hline $\begin{array}{l}\text { Mecklenburg- } \\
\text { Vorpom- } \\
\text { mern }\end{array}$ & 0,01 & 0,83 & 1,45 & 0,54 & 0,19 & 0,62 & 0,68 & 0,25 \\
\hline Niedersachsen & 0,17 & 1,09 & 2,43 & 0,38 & $-0,03$ & 0,53 & 0,52 & 0,08 \\
\hline $\begin{array}{l}\text { Nordrhein- } \\
\text { Westfalen }\end{array}$ & 0,07 & 0,47 & 0,31 & 0,07 & 0,00 & 0,55 & 0,37 & 0,08 \\
\hline $\begin{array}{l}\text { Rheinland- } \\
\text { Pfalz }\end{array}$ & $-0,15$ & 0,69 & 0,69 & 0,18 & $-0,03$ & 0,75 & 0,74 & 0,19 \\
\hline Saarland & $-0,14$ & 1,23 & 2,16 & 0,16 & $-0,23$ & 1,12 & 2,09 & 0,16 \\
\hline Sachsen & $-0,01$ & 0,68 & 0,68 & 0,18 & 0,10 & 0,61 & 0,55 & 0,15 \\
\hline $\begin{array}{l}\text { Sachsen- } \\
\text { Anhalt }\end{array}$ & $-0,04$ & 0,95 & 1,69 & 0,38 & 0,06 & 0,82 & 1,16 & 0,26 \\
\hline $\begin{array}{l}\text { Schleswig- } \\
\text { Holstein }\end{array}$ & $-0,02$ & 0,89 & 1,11 & 0,43 & 0,01 & 0,71 & 0,87 & 0,34 \\
\hline Thüringen & 0,21 & 0,73 & 0,86 & 0,17 & 0,16 & 1,00 & 1,46 & 0,29 \\
\hline \multirow[t]{2}{*}{ Bund } & 0,06 & 0,32 & 0,19 & 0,05 & 0,01 & 0,27 & 0,16 & 0,04 \\
\hline & \multicolumn{4}{|c|}{$\begin{array}{l}\text { Erste vs. dritte Originärberechnung } \\
\text { 2000-2015 }\end{array}$} & \multicolumn{4}{|c|}{$\begin{array}{l}\text { Erste Fortschreibung vs. dritte Origi- } \\
\text { närberechnung } \\
2000-2015\end{array}$} \\
\hline $\begin{array}{l}\text { Baden- } \\
\text { Württem- } \\
\text { berg }\end{array}$ & 0,13 & 0,29 & 0,11 & 0,01 & 0,12 & 1,06 & 1,58 & 0,14 \\
\hline Bayern & 0,12 & 0,36 & 0,20 & 0,06 & 0,60 & 0,90 & 1,46 & 0,33 \\
\hline Berlin & 0,09 & 0,52 & 0,34 & 0,12 & 0,15 & 1,29 & 1,90 & 0,44 \\
\hline Brandenburg & 0,34 & 0,58 & 0,44 & 0,13 & 0,73 & 1,14 & 2,36 & 0,74 \\
\hline Bremen & 0,24 & 0,57 & 0,47 & 0,04 & $-0,18$ & 1,46 & 4,18 & 0,41 \\
\hline Hamburg & 0,31 & 0,77 & 1,16 & 0,24 & $-0,38$ & 1,31 & 2,82 & 0,54 \\
\hline Hessen & 0,03 & 0,47 & 0,41 & 0,11 & $-0,15$ & 1,05 & 1,59 & 0,31 \\
\hline $\begin{array}{l}\text { Mecklenburg- } \\
\text { Vorpom- } \\
\text { mern }\end{array}$ & 0,30 & 0,51 & 0,38 & 0,13 & 0,45 & 1,05 & 1,68 & 0,56 \\
\hline Niedersachsen & 0,21 & 0,36 & 0,24 & 0,04 & 0,24 & 0,91 & 1,14 & 0,19 \\
\hline $\begin{array}{l}\text { Nordrhein- } \\
\text { Westfalen }\end{array}$ & $-0,09$ & 0,42 & 0,31 & 0,06 & $-0,02$ & 0,47 & 0,41 & 0,09 \\
\hline
\end{tabular}


Tab. 1 (Fortsetzung)

\begin{tabular}{lcccccccc}
\hline $\begin{array}{l}\text { Rheinland- } \\
\text { Pfalz }\end{array}$ & 0,09 & 0,23 & 0,11 & 0,03 & $-0,10$ & 0,63 & 0,66 & 0,16 \\
Saarland & 0,02 & 0,57 & 0,64 & 0,04 & 0,04 & 1,24 & 2,12 & 0,15 \\
Sachsen & 0,37 & 0,47 & 0,49 & 0,13 & 0,35 & 0,81 & 1,03 & 0,22 \\
$\begin{array}{l}\text { Sachsen- } \\
\text { Anhalt }\end{array}$ & 0,18 & 0,33 & 0,17 & 0,03 & 0,06 & 1,17 & 2,30 & 0,43 \\
$\begin{array}{l}\text { Schleswig- } \\
\text { Holstein }\end{array}$ & $-0,01$ & 0,28 & 0,18 & 0,07 & $-0,06$ & 1,05 & 1,73 & 0,50 \\
$\begin{array}{l}\text { Thüringen } \\
\text { Bund }\end{array}$ & 0,34 & 0,40 & 0,25 & 0,04 & 0,63 & 1,01 & 1,46 & 0,24 \\
\hline
\end{tabular}

- Die mittlere Revision (MR), berechnet als der Mittelwert aller Differenzen zwischen Revision und Vergleichsberechnung. Sie ist ein Maß der Verzerrung.

- Die mittlere absolute Revision (MAR), berechnet als der Mittelwert aller absoluten Differenzen zwischen Revision und Vergleichsberechnung. Sie wird in Prozentpunkten angegeben und veranschaulicht das Ausmaß der Revisionen unter Zugrundelegung einer linearen Verlustfunktion.

- Die analog berechnete mittlere quadratische Revision (MQR) impliziert hingegen eine quadratische Verlustfunktion, gewichtet also große Revisionen höher. Sind die Revisionen unverzerrt, kann die Wurzel aus der MQR als Streuung der Revisionen interpretiert und zur Berechnung von Revisionsintervallen herangezogen werden.

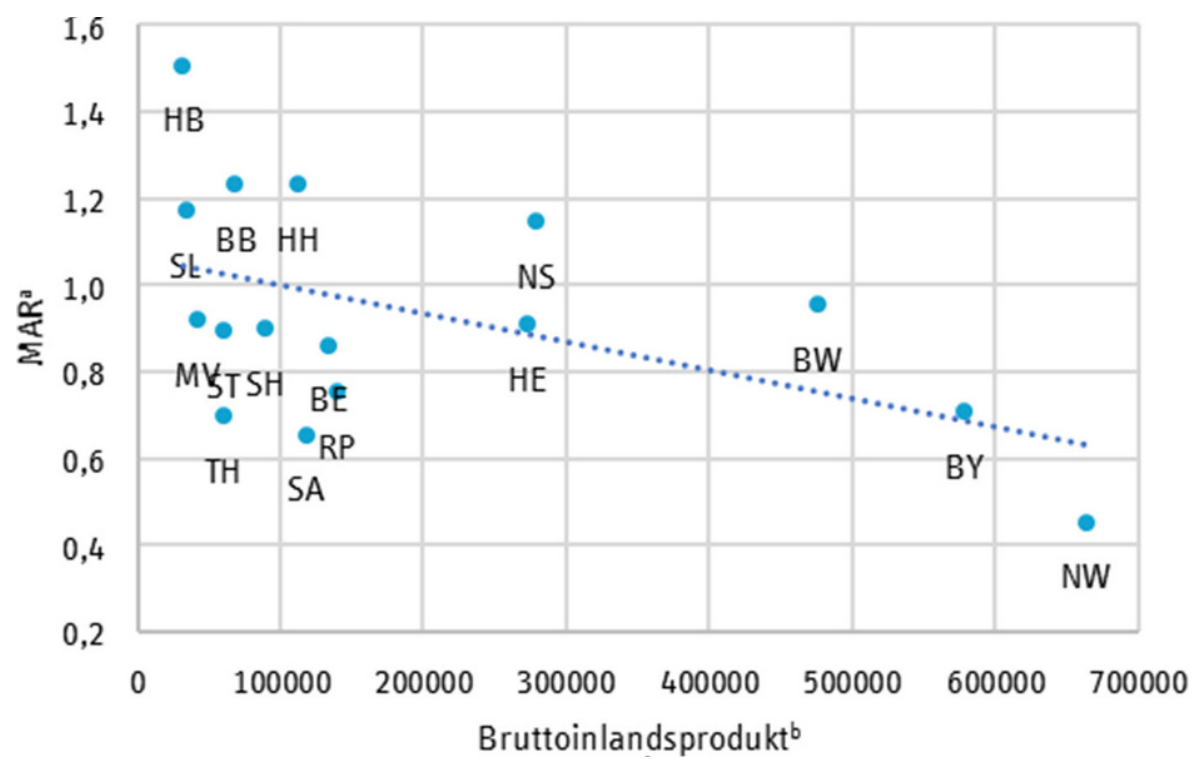

Abb. 1 Mittlere absolute Revision ${ }^{\mathrm{a}}$ und Höhe des BIP ${ }^{\mathrm{b}}$. (Eigene Berechnungen: ${ }^{\text {a} E r s t e ~ O r i g i n a ̈ r b e r e c h-~}$

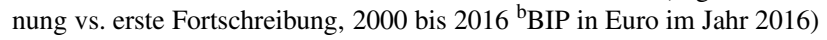


- Die Noise-to-signal rate (NSR) setzt die MQR in Relation zur Varianz der Originärberechnung. Sie kann als Maß für den Informationsgehalt der jeweiligen Veröffentlichungen interpretiert werden. Ist die NSR größer als 1, ist der Informationsgehalt der vorläufigen Daten gering.

Um einen Eindruck zu vermitteln, wie weit die VGR der Ländern Revisionen der gesamtdeutschen VGR widerspiegeln, werden alle Prüfmaße auch für das in den jeweiligen Tabellen der VGR der Länder veröffentlichte Bundesergebnis angegeben.

Ausgangspunkt der Berechnungen der VGR der Länder sind die Nominalwerte; sie werden daher als erstes betrachtet. Tab. 1 zeigt, dass die Revisionen von der ersten Fortschreibung bis zur ersten Originärberechnung beträchtlich sind und die beim Bund bei weitem übersteigen. Die mittlere absolute Revision beträgt bei fünf Ländern mehr als einen Prozentpunkt. Am geringsten ist sie bei Nordrhein-Westfalen, aber auch hier ist sie stärker als beim gesamtdeutschen BIP. Tendenziell nimmt das Ausmaß der Revisionen mit der Größe der Länder ab. Dies überrascht insofern nicht, als kleine Länder stark durch einzelne Branchen oder gar Unternehmen geprägt sind. Strikt ist dieser Zusammenhang aber nicht, auch die Revisionen für Rheinland-Pfalz, Sachsen und Thüringen sind gering (Abb. 1). ${ }^{7}$

Im Vergleich von zweiter Fortschreibung und erster Originärberechnung sind die Revisionsmaße nur unwesentlich kleiner als bei der ersten Fortschreibung. Dies verdeutlicht, dass die größeren Veränderungen mit der ersten Originärberechnung vorgenommen werden, weil erst dann die meisten Datenquellen eingearbeitet werden können. Die zwischen der ersten und der dritten Originärberechnung vorgenommenen Änderungen sind bei allen Ländern geringer als die der ersten Revisionsphase. Die mittlere absolute Revision liegt schwerpunktmäßig bei 0,5 Prozentpunkten; am niedrigsten ist auch hier der Wert von Rheinland-Pfalz, am größten der für Hamburg. Sie ist aber in allem Ländern größer als beim Bund.

Über den gesamten Revisionszyklus gesehen fallen die Revisionen im Durchschnitt nur unwesentlich größer, bei Niedersachsen und Rheinland-Pfalz sogar etwas geringer aus als in der ersten Phase, also bis zur Vorlage der ersten Originärberechnung. Dies zeigt, dass die im Überarbeitungsprozess vorgenommenen Revisionen nicht alle in die gleiche Richtung gehen, sondern dass in vielen Fällen die vorläufigen Raten um die endgültigen schwanken.

Die mittlere quadratische Revision ist in den meisten Ländern nur wenig größer als die mittlere absolute. Dies deutet darauf hin, dass alle Jahre in ähnlichem Maße von Revisionen betroffen sind. Die auffälligste Ausnahme ist hier Bremen, aber auch bei Niedersachsen, dem Saarland und Brandenburg treten größere Unterschiede zwischen beiden Kennziffern auf. Dies bedeutet, dass in diesen Ländern die Revisionen stark von großen Änderungen in einzelnen Jahren geprägt sind. Erfreulich ist, dass die noise to signal ratio in allen Ländern und für alle Vergleiche kleiner als eins ist. Bereits die ersten Veröffentlichungen haben danach zumindest einen gewissen Informationsgehalt bezüglich der finalen Werte.

\footnotetext{
7 Regressiert man die MAR auf das BIP im Jahr 2016, so erhält man einen negativen Zusammenhang, der nur auf dem $90 \%$-Niveau signifikant ist.
} 
Tab. 2 Revisionen der Vorjahresraten des realen BIP der Länder 2000-2017. (Quelle: Eigene Berechnungen nach Angaben des Arbeitskreises VGR der Länder)

\begin{tabular}{|c|c|c|c|c|c|c|c|c|}
\hline & \multicolumn{4}{|c|}{$\begin{array}{l}\text { Erste Fortschreibung vs. erste Originärbe- } \\
\text { rechnung } \\
2000-2017\end{array}$} & \multicolumn{4}{|c|}{$\begin{array}{l}\text { Erste Fortschreibung vs. dritte Originärbe- } \\
\text { rechnung } \\
2000-2015\end{array}$} \\
\hline & MR & MAR & MQR & NSR & MR & MAR & MQR & NSR \\
\hline $\begin{array}{l}\text { Baden- } \\
\text { Württem- } \\
\text { berg }\end{array}$ & $-0,15$ & 0,93 & 1,19 & 0,11 & $-0,05$ & 0,92 & 1,26 & 0,10 \\
\hline Bayern & 0,43 & 0,64 & 0,81 & 0,21 & 0,63 & 0,80 & 1,18 & 0,25 \\
\hline Berlin & 0,16 & 0,89 & 1,23 & 0,31 & 0,07 & 1,14 & 1,77 & 0,35 \\
\hline Brandenburg & 0,41 & 1,17 & 2,00 & 0,71 & 0,70 & 1,18 & 2,33 & 0,89 \\
\hline Bremen & $-0,51$ & 1,20 & 2,46 & 0,37 & $-0,22$ & 1,36 & 3,18 & 0,38 \\
\hline Hamburg & $-0,57$ & 1,14 & 2,11 & 0,83 & $-0,08$ & 1,35 & 2,72 & 0,51 \\
\hline Hessen & $-0,42$ & 0,79 & 1,02 & 0,21 & $-0,38$ & 0,77 & 1,09 & 0,18 \\
\hline $\begin{array}{l}\text { Mecklenburg- } \\
\text { Vorpom- } \\
\text { mern }\end{array}$ & 0,35 & 0,81 & 1,27 & 0,78 & 0,36 & 0,87 & 1,11 & 0,61 \\
\hline Niedersachsen & 0,07 & 0,96 & 2,09 & 0,41 & 0,09 & 0,82 & 1,09 & 0,22 \\
\hline $\begin{array}{l}\text { Nordrhein- } \\
\text { Westfalen }\end{array}$ & 0,05 & 0,57 & 0,38 & 0,10 & 0,16 & 0,60 & 0,59 & 0,16 \\
\hline $\begin{array}{l}\text { Rheinland- } \\
\text { Pfalz }\end{array}$ & $-0,27$ & 0,64 & 0,61 & 0,16 & 0,00 & 0,60 & 0,56 & 0,13 \\
\hline Saarland & $-0,23$ & 1,06 & 1,94 & 0,15 & $-0,15$ & 1,11 & 1,77 & 0,13 \\
\hline Sachsen & 0,17 & 0,57 & 0,49 & 0,17 & 0,38 & 0,75 & 0,90 & 0,24 \\
\hline $\begin{array}{l}\text { Sachsen- } \\
\text { Anhalt }\end{array}$ & 0,05 & 1,02 & 1,86 & 0,46 & 0,19 & 1,08 & 1,94 & 0,44 \\
\hline $\begin{array}{l}\text { Schleswig- } \\
\text { Holstein }\end{array}$ & 0,14 & 0,82 & 0,96 & 0,52 & 0,08 & 0,99 & 1,52 & 0,51 \\
\hline Thüringen & 0,29 & 0,67 & 0,76 & 0,16 & 0,58 & 0,95 & 1,32 & 0,24 \\
\hline Bund & 0,03 & 0,20 & 0,06 & 0,02 & 0,16 & 0,34 & 0,18 & 0,04 \\
\hline
\end{tabular}

Die mittlere Revision schließlich lässt keine signifikanten Verzerrungen erkennen. Etwa bei der Hälfte der Länder ist die als erste veröffentlichte Veränderungsrate im Durchschnitt zu hoch (d.h. MR ist negativ). Auffällig ist die Verzerrung aber nur bei Bayern und Hamburg, wo sie auf dem $90 \%$-Niveau signifikant von Null verschieden ist.

Tab. 2 enthält die gleichen Revisionsmaße für das reale BIP, wobei aus Platzgründen nur zwei Vergleiche dargestellt werden. Die Maße unterscheiden sich von denen beim nominalen BIP nur unwesentlich. Korreliert man länderweise die Revisionen der Zuwachsraten des nominalen und des realen BIP, so liegt der Korrelationskoeffizient zumeist über 0,9. Ausnahmen sind hier Hamburg und Mecklenburg-Vorpommern sowie - weniger ausgeprägt - Schleswig-Holstein. Die hohe Parallelität könnte man so verstehen, dass das nominale BIP bei allen Revisionsschritten mit einem wenig veränderten Preisindex deflationiert wird.

Diese Vermutung lässt sich aber nicht bestätigen. Vielmehr sind die impliziten Deflatoren erheblichen Revisionen unterworfen (Tab. 3). Bei den großen Bundesländern Nordrhein-Westfalen, Bayern und Baden-Württemberg, deren Wirtschafts- 
strukturen stärker der gesamtdeutschen ähneln, entspricht die Revision in etwa der beim gesamtdeutschen Deflator. Bei den kleineren Bundesländern sind die Revisionen erheblich größer, bei Hamburg und Mecklenburg-Vorpommern übersteigen sie über den gesamten Revisionsprozess gesehen sogar die Varianz des Deflators, d.h. die noise to signal-ratio ist größer als 1 .

Da laut Methodenbeschreibung auf der untersten Berechnungsebene gleiche Deflatoren für alle Länder verwendet werden, können sich Abweichungen der Revisionen auf Länderebene von denen für den Bund nur durch das Zusammenwirken von Unterschieden in der sektoralen Zusammensetzung der Wertschöpfung und von gegenläufigen Revisionen der sektoralen Deflatoren auf Bundesebene ergeben. Dass solche Kompositionseffekte bei kleineren Ländern aufgrund deren spezialisierten Wirtschaftsstrukturen stärker durchschlagen können als in großen, überrascht deshalb nicht.

Um die Hintergründe der Revisionen besser zu verstehen, bietet es sich an, die Änderungen beim realen BIP aufzuspalten in Revision der Zunahme des Arbeitsin-

Tab. 3 Revisionen der Vorjahresveränderungen der BIP-Deflatoren der Länder 2000-2017. (Quelle: Eigene Berechnungen nach Angaben des Arbeitskreises VGR der Länder)

\begin{tabular}{|c|c|c|c|c|c|c|c|c|}
\hline & \multicolumn{4}{|c|}{$\begin{array}{l}\text { Erste Fortschreibung vs. erste Originärbe- } \\
\text { rechnung } \\
2000-2017\end{array}$} & \multicolumn{4}{|c|}{$\begin{array}{l}\text { Erste Fortschreibung vs. dritte Originärbe- } \\
\text { rechnung } \\
2000-2015\end{array}$} \\
\hline & MR & MAR & MQR & NSR & MR & MAR & MQR & NSR \\
\hline $\begin{array}{l}\text { Baden- } \\
\text { Württem- } \\
\text { berg }\end{array}$ & 0,12 & 0,24 & 0,09 & 0,19 & 0,12 & 0,24 & 0,09 & 0,21 \\
\hline Bayern & 0,01 & 0,23 & 0,09 & 0,16 & $-0,08$ & 0,27 & 0,12 & 0,22 \\
\hline Berlin & 0,01 & 0,39 & 0,23 & 0,51 & 0,08 & 0,49 & 0,35 & 0,97 \\
\hline Brandenburg & $-0,13$ & 0,41 & 0,23 & 0,40 & $-0,14$ & 0,42 & 0,26 & 0,46 \\
\hline Bremen & $-0,14$ & 0,46 & 0,60 & 0,67 & $-0,10$ & 0,47 & 0,35 & 0,73 \\
\hline Hamburg & 0,02 & 1,04 & 1,92 & 0,94 & $-0,21$ & 0,80 & 1,25 & 1,07 \\
\hline Hessen & 0,23 & 0,43 & 0,26 & 0,41 & 0,17 & 0,53 & 0,39 & 0,52 \\
\hline $\begin{array}{l}\text { Mecklenburg- } \\
\text { Vorpom- } \\
\text { mern }\end{array}$ & $-0,20$ & 0,74 & 0,96 & 0,80 & $-0,06$ & 0,74 & 0,76 & 1,09 \\
\hline Niedersachsen & $-0,03$ & 0,41 & 0,25 & 0,92 & $-0,05$ & 0,40 & 0,22 & 0,83 \\
\hline $\begin{array}{l}\text { Nordrhein- } \\
\text { Westfalen }\end{array}$ & 0,01 & 0,25 & 0,11 & 0,36 & $-0,10$ & 0,24 & 0,10 & 0,31 \\
\hline $\begin{array}{l}\text { Rheinland- } \\
\text { Pfalz }\end{array}$ & 0,03 & 0,25 & 0,12 & 0,26 & $-0,02$ & 0,15 & 0,04 & 0,08 \\
\hline Saarland & 0,09 & 0,43 & 0,27 & 0,63 & 0,05 & 0,44 & 0,29 & 0,78 \\
\hline Sachsen & $-0,16$ & 0,36 & 0,17 & 0,26 & $-0,05$ & 0,37 & 0,18 & 0,38 \\
\hline $\begin{array}{l}\text { Sachsen- } \\
\text { Anhalt }\end{array}$ & $-0,10$ & 0,39 & 0,19 & 0,47 & $-0,13$ & 0,39 & 0,22 & 0,43 \\
\hline $\begin{array}{l}\text { Schleswig- } \\
\text { Holstein }\end{array}$ & $-0,11$ & 0,47 & 0,42 & 0,67 & $-0,19$ & 0,46 & 0,35 & 0,82 \\
\hline Thüringen & $-0,11$ & 0,31 & 0,14 & 0,27 & $-0,04$ & 0,43 & 0,23 & 0,57 \\
\hline Bund & 0,02 & 0,21 & 0,08 & 0,24 & $-0,03$ & 0,25 & 0,09 & 0,29 \\
\hline
\end{tabular}


put, gemessen am Arbeitsvolumen, und der Produktivität, gemessen an der realen BWS je Arbeitsstunde. Da allerdings für die Jahre vor 2005 keine Angaben zum Arbeitsvolumen vorliegen, stützen sich diese Berechnungen auf eine kurze Datenreihe. Betrachtet werden hier nur die Revisionen der ersten Originärberechnung gegenüber der ersten Fortschreibung, denn die Einbeziehung der dritten Originärberechnung würde den Untersuchungszeitraum weiter verkürzen.

Die Beiträge des Arbeitsinputs und der Produktivität zur Revision des realen BIP unterscheiden sich deutlich (Tab. 4). Die Zunahme des Arbeitsvolumens wird im Durchschnitt im Bundesgebiet insgesamt wie in den Ländern zwar abwärtsrevidiert (MR ist negativ), die durchschnittlichen Revisionen sind aber auf der Ebene der Länder im Allgemeinen nur wenig größer als beim Bund, und der Unterscheid zwischen kleinen und großen Ländern ist nicht allzu gravierend. Die Noise-to-signalRatio ist durchgängig deutlich kleiner als 1. Geänderte Zuwächse beim Arbeitsinput sind demnach nicht die vorwiegende Ursache für Revisionen der BWS bzw. des BIP. Zudem sind die Revisionen beim realen BIP und beim Arbeitsvolumen in den meisten Ländern und beim Bund nur schwach korreliert (Tab. 5). Eine signifikante positive Korrelation findet man nur bei Berlin und Mecklenburg-Vorpommern, während man bei Thüringen und - weniger ausgeprägt - Sachsen und Bremen sogar eine signifikante negative Korrelation findet.

Tab. 4 Revisionen der Vorjahresveränderungen von Arbeitsvolumen und Produktivität 2005-2017, Erste Originärberechnung gegenüber erster Fortschreibung. (Quelle: Eigene Berechnungen nach Angaben des Arbeitskreises VGR der Länder)

\begin{tabular}{lllllllll}
\hline & \multicolumn{1}{l}{ Arbeitsvolumen } & \multicolumn{7}{l}{ Produktiviät } \\
& MR & MAR & MQR & NSR & MR & MAR & MQR & NSR \\
\hline Baden-Württemberg & $-0,20$ & 0,31 & 0,14 & 0,04 & 0,13 & 0,78 & 1,03 & 0,18 \\
Bayern & $-0,07$ & 0,34 & 0,28 & 0,14 & 0,55 & 0,83 & 1,81 & 0,96 \\
Berlin & $-0,28$ & 0,53 & 0,47 & 0,50 & 0,77 & 0,82 & 1,11 & 1,25 \\
Brandenburg & $-0,20$ & 0,60 & 0,55 & 0,51 & 0,45 & 1,37 & 2,48 & 1,04 \\
Bremen & $-0,16$ & 0,46 & 0,33 & 0,17 & $-0,41$ & 1,56 & 3,85 & 0,94 \\
Hamburg & $-0,04$ & 0,41 & 0,26 & 0,27 & $-0,51$ & 1,19 & 1,88 & 1,45 \\
Hessen & $-0,24$ & 0,39 & 0,20 & 0,15 & $-0,13$ & 0,72 & 0,71 & 0,31 \\
Mecklenburg-Vor- & $-0,19$ & 0,63 & 0,90 & 0,50 & 0,52 & 1,10 & 1,64 & 1,02 \\
pommern & & & & & & & & \\
Niedersachsen & $-0,13$ & 0,36 & 0,16 & 0,13 & 0,47 & 1,14 & 2,73 & 0,70 \\
Nordrhein-Westfalen & $-0,11$ & 0,24 & 0,09 & 0,05 & 0,29 & 0,63 & 0,51 & 0,41 \\
Rheinland-Pfalz & $-0,08$ & 0,26 & 0,13 & 0,07 & $-0,10$ & 0,78 & 0,82 & 0,50 \\
Saarland & $-0,32$ & 0,43 & 0,26 & 0,10 & $-0,08$ & 0,99 & 1,60 & 0,20 \\
Sachsen & $-0,15$ & 0,46 & 0,33 & 0,23 & 0,44 & 0,86 & 1,01 & 0,77 \\
Sachsen-Anhalt & $-0,24$ & 0,42 & 0,28 & 0,21 & 0,16 & 1,35 & 2,74 & 0,88 \\
Schleswig-Holstein & $-0,24$ & 0,32 & 0,18 & 0,14 & 0,39 & 0,79 & 1,09 & 0,95 \\
Thüringen & $-0,18$ & 0,41 & 0,27 & 0,13 & 0,48 & 0,98 & 1,66 & 0,74 \\
Bund & $-0,15$ & 0,21 & 0,08 & 0,05 & 0,27 & 0,32 & 0,15 & 0,10 \\
\hline
\end{tabular}


Tab. 5 Korrelation der Revisionen der Veränderungsraten von BIP, Arbeitsvolumen und Produktivität 2005-2017, Erste Originärberechnung gegenüber erster Fortschreibung. (Quelle: Eigene Berechnungen nach Angaben des Arbeitskreises VGR der Länder)

\begin{tabular}{llll}
\hline & $\begin{array}{l}\text { BIP vs Arbeitsvolu- } \\
\text { men }\end{array}$ & $\begin{array}{l}\text { BIP vs Produktivi- } \\
\text { tät }\end{array}$ & $\begin{array}{l}\text { Arbeitsvolumen vs Produkti- } \\
\text { vität }\end{array}$ \\
\hline Baden-Württemberg & 0,098 & 0,901 & $-0,313$ \\
Bayern & $-0,379$ & 0,939 & $-0,649$ \\
Berlin & 0,753 & 0,837 & 0,304 \\
Brandenburg & $-0,046$ & 0,899 & $-0,465$ \\
Bremen & $-0,446$ & 0,971 & $-0,636$ \\
Hamburg & 0,077 & 0,932 & $-0,272$ \\
Hessen & 0,215 & 0,903 & $-0,180$ \\
Mecklenburg-Vor- & 0,476 & 0,723 & $-0,248$ \\
pommern & & & \\
Niedersachsen & 0,238 & 0,978 & 0,056 \\
Nordrhein-Westfalen & $-0,087$ & 0,887 & $-0,436$ \\
Rheinland-Pfalz & $-0,255$ & 0,913 & $-0,568$ \\
Saarland & $-0,024$ & 0,948 & $-0,321$ \\
Sachsen & $-0,410$ & 0,841 & $-0,804$ \\
Sachsen-Anhalt & $-0,263$ & 0,964 & $-0,487$ \\
Schleswig-Holstein & 0,135 & 0,908 & $-0,251$ \\
Thüringen & $-0,604$ & 0,930 & $-0,830$ \\
Bund & 0,240 & 0,549 & $-0,435$ \\
Alle Länder & 0,009 & 0,915 & $-0,367$ \\
\hline Gepoolte & & & \\
\hline
\end{tabular}

${ }^{\mathrm{a} G e p o o l t e ~ D a t e n}$

Deutlich anders ist das Bild bei der Produktivität. Hier sind die Revisionen deutlich ausgeprägter als die beim BIP ${ }^{8}$. Bei der Hälfte der Länder beträgt die mittlere absolute Revision des Produktivitätswachstum mehr als einen Prozentpunkt. Bei vier Ländern ist die Noise-to signal-ratio größer als eins, und bei weiteren zwei Ländern - darunter mit Bayern eines der großen Länder - liegt sie nur sehr knapp darunter. Dies zeigt, dass Revisionen beim BIP fast vollständig auf die Produktivität durchschlagen. Die Korrelation der Revisionen beider Größen übersteigt in der Mehrzahl der Länder den Wert von 0,9; beim Bund beträgt sie nur 0,55.

\subsection{Rangstabilität der Angaben}

Da die VGR der Länder häufig verwendet werden, um Länder hinsichtlich ihres Wachstumstempos zu vergleichen, wird in einem weiteren Analyseschritt untersucht, wie sich die Rangfolge der Länder beim BIP-Wachstum im Zuge der verschiedenen Revisionsrunden ändert.

$\mathrm{Ob}$ es ein Kriterium für die Qualität der VGR der Länder ist, wenn sich die Rangfolge der Länder bezüglich der Zuwächse des BIP im Zuge der Revisionen nicht ändert, lässt sich kontrovers diskutieren. Für die Nutzer, insbesondere die Po-

\footnotetext{
${ }^{8}$ Dies gilt auch, wenn man die Revisionen des BIP im gleichen Zeitraum betrachtet.
} 


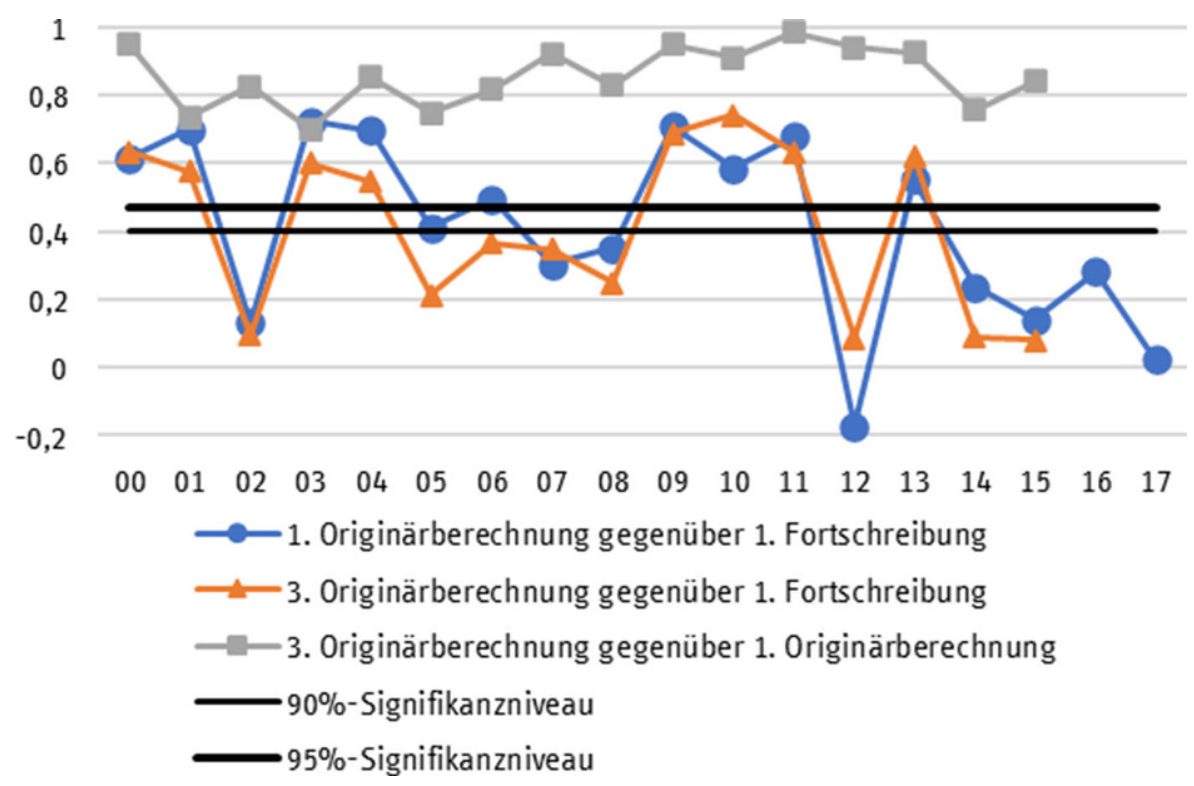

Abb. 2 Ränge der Länder bezüglich der Zunahme des BIP nach unterschiedlichen Revisionsständen der VGR der Länder 2000 bis 2017, Rangkorrelationskoeffizienten. (Eigene Berechnungen)

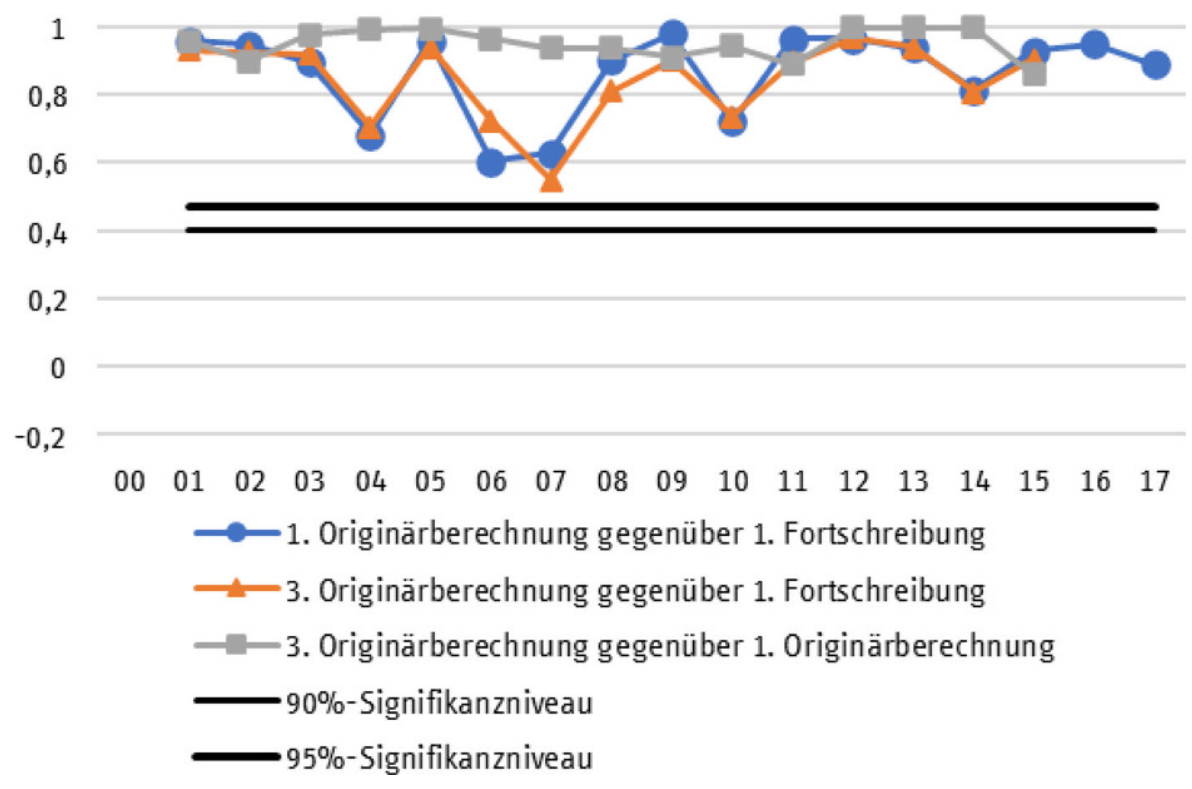

Abb. 3 Ränge der Länder bezüglich der Zunahme der Erwerbstätigkeit nach unterschiedlichen Revisionsständen der VGR der Länder 2000 bis 2016, Rangkorrelationskoeffizienten. (Eigene Berechnungen) 
litik, ist es gleichwohl ein wichtiges Kriterium, wird doch eine im Vergleich zum Bund überdurchschnittliche Zuwachsrate gerne als Ausweis einer erfolgreichen Politik angesehen, und umgekehrt eine unterdurchschnittliche Rate als Hinweis auf Handlungsbedarf verstanden. Da hilft es wenig, wenn nach Abschluss des Revisionsprozesses nach vier Jahren, also nach einer Zeit, die oft der Dauer einer Legislaturperiode entspricht, die Öffentlichkeit erfährt, dass es anders war.

Als Maß der Rangstabilität werden hier Rangkorrelationskoeffizienten verwendet. Abb. 2 zeigt, dass die Ränge der Länder bezüglich der Zunahme des BIP gemäß der ersten Fortschreibung in den meisten Jahren wenig mit Rangfolge bei den Originärberechnungen zu tun haben. In etwa der Hälfte der betrachteten Jahre - die Ergebnisse unterscheiden sich leicht, je nachdem, ob man die erste oder die dritte Originärberechnung als Maßstab heranzieht - besteht zwischen den sich aus der Erstveröffentlichung ergebenden Rangfolgen und den später veröffentlichten kein statistisch signifikanter Zusammenhang. Der Vergleich von der dritten mit der ersten Originärberechnung fällt hingegen günstiger aus. Zwischen diesen beiden Berechnungsstufen ändert sich die Rangfolge der Länder kaum.

Bei den Erwerbstätigen zeigt sich ein anderes Bild. Hier ändert sich die Rangfolge der Länder im Zuge des Revisionsprozesses nur wenig; der Rangkorrelationskoeffizient ist in allen Fällen signifikant von Null verschieden (Abb. 3). Dies ist - neben der generell geringeren Revisionsanfälligkeit - ein zweiter Hinweis, dass die Erwerbstätigkeit ein möglicherweise aussagekräftigerer Indikator der wirtschaftlichen Entwicklung von Bundesländern als das BIP ist.

\section{Revisionen auf der Ebene von Wirtschaftszweigen}

Da die VGR der Länder ausgehend von der Ebene der Wirtschaftszweige erstellt wird, ist es von Interesse, wie weit die einzelnen Branchen zu den Revisionen auf gesamtwirtschaftlicher Ebene beitragen. Angaben zur Wertschöpfung liefert die VGR der Länder derzeit für 20 Wirtschaftszweige (Abschnitte der Wirtschaftszweigesystematik WZ 2008). Allerdings findet man nicht für alle lange Zeitreihen, zum einen weil im Zuge der Generalrevision 2011 die Wirtschaftszweigesystematik geändert wurde, zum anderen weil Angaben für manche Wirtschaftszweige mit Verzögerung anfallen. Zudem liegen Angaben zum Arbeitsvolumen ohnehin für eine geringere Zahl von Wirtschaftszweigen vor. Daher beschränken sich die folgenden Ausführungen auf das Verarbeitende Gewerbe, das Baugewerbe und die Gesamtheit der Dienstleistungssektoren.

Im Verarbeitenden Gewerbe werden die Zuwachsraten der nominalen und der realen Bruttowertschöpfung für nahezu alle Länder stärker revidiert als für den Bund (Tab. 6). ${ }^{9}$ Gleichwohl ist die Noise to signal ratio in allen Fällen deutlich kleiner als 1, besitzt also bereits die erste Veröffentlichung einen gewissen Informationsgehalt. In der Grundtendenz gilt auch hier, dass die Revisionen bei großen Ländern geringer ausfallen als bei kleinen. Auffällig sind aber die vergleichsweise große Revision

\footnotetext{
9 Aus Platzgründen werden hier nur MAR und NSR und nur ein Vergleich der ersten Originärberechnung mit der ersten Fortschreibung ausgewiesen. Zusätzliche Angaben stehen auf Anfrage zur Verfügung.
} 
Tab. 6 Revisionen der VGR der Länder für das Verarbeitende Gewerbe. Veränderungen gegenüber dem Vorjahr; Erste Originärberechnung gegenüber erster Fortschreibung. (Quelle: Eigene Berechnungen nach Angaben des Arbeitskreises VGR der Länder)

\begin{tabular}{|c|c|c|c|c|c|c|c|c|}
\hline & \multicolumn{2}{|c|}{$\begin{array}{l}\text { BWS } \\
\text { nominal }^{\mathrm{a}}\end{array}$} & \multicolumn{2}{|l|}{$\begin{array}{l}\text { BWS } \\
\text { real }^{\mathrm{a}}\end{array}$} & \multicolumn{2}{|c|}{ Arbeitsvolumen $^{\mathrm{b}}$} & \multicolumn{2}{|c|}{ Arbeitsproduktivität } \\
\hline & MAR & NSR & MAR & NSR & MAR & NSR & MAR & NSR \\
\hline Baden-Württemberg & 1,9 & 0,11 & 2,7 & 0,14 & 0,4 & 0,02 & 2,3 & 0,21 \\
\hline Bayern & 2,3 & 0,44 & 2,5 & 0,22 & 0,4 & 0,03 & 2,8 & 0,45 \\
\hline Berlin & 2,6 & 0,57 & 3,3 & 0,57 & 0,7 & 0,14 & 3,1 & 0,65 \\
\hline Brandenburg & 3,4 & 0,48 & 4,5 & 0,59 & 0,7 & 0,05 & 5,5 & 0,98 \\
\hline Bremen & 5,6 & 0,58 & 6,4 & 0,68 & 1,3 & 0,39 & 9,0 & 1,08 \\
\hline Hamburg & 3,5 & 0,32 & 4,9 & 0,49 & 0,8 & 0,32 & 6,1 & 0,73 \\
\hline Hessen & 2,6 & 0,33 & 3,7 & 0,36 & 0,5 & 0,03 & 4,2 & 0,54 \\
\hline $\begin{array}{l}\text { Mecklenburg-Vor- } \\
\text { pommern }\end{array}$ & 3,9 & 0,69 & 4,5 & 0,63 & 1,4 & 0,51 & 5,3 & 0,92 \\
\hline Niedersachsen & 4,8 & 0,57 & 5,5 & 0,54 & 0,7 & 0,11 & 7,2 & 0,71 \\
\hline $\begin{array}{l}\text { Nordrhein-Westfa- } \\
\text { len }\end{array}$ & 2,5 & 0,26 & 2,6 & 0,21 & 0,4 & 0,02 & 2,8 & 0,38 \\
\hline Rheinland-Pfalz & 3,1 & 0,57 & 2,0 & 0,15 & 0,5 & 0,08 & 2,6 & 0,28 \\
\hline Saarland & 3,4 & 0,14 & 4,5 & 0,24 & 0,6 & 0,03 & 3,4 & 0,20 \\
\hline Sachsen & 3,1 & 0,36 & 3,3 & 0,42 & 0,5 & 0,03 & 3,4 & 0,45 \\
\hline Sachsen-Anhalt & 3,9 & 0,46 & 4,5 & 0,40 & 0,8 & 0,14 & 6,0 & 0,61 \\
\hline Schleswig-Holstein & 3,4 & 0,56 & 3,6 & 0,46 & 0,7 & 0,18 & 3,7 & 0,64 \\
\hline Thüringen & 2,0 & 0,16 & 2,8 & 0,22 & 0,6 & 0,06 & 3,6 & 0,44 \\
\hline Bund & 1,9 & 0,13 & 2,2 & 0,16 & 0,3 & 0,01 & 2,6 & 0,28 \\
\hline
\end{tabular}

a2001 bis 2017

b2007 bis 2017

${ }^{\mathrm{c}}$ Reale Bruttowertschöpfung je Erwerbstätigenstunde; 2007 bis 2017

beim gemessen am BIP viertgrößten Land Niedersachsen. Hier schlägt u.a. die in der Einleitung angesprochene kräftige Revision im Jahr 2016 zu Buche.

Die Revisionen des Arbeitsvolumens sind wie in der Gesamtwirtschaft auch beim Verarbeitenden Gewerbe deutlich geringer als bei der Wertschöpfung, sowohl gemessen an MAR als auch an NSR. Dies impliziert große Revisionen der Arbeitsproduktivität; hier übersteigt die NSR in Bremen den Wert von 1, und sie liegt in Brandenburg und in Mecklenburg-Vorpommern nur knapp darunter.

Massive Zweifel an der Verlässlichkeit der ersten Fortschreibung ergibt die Revisionsanalyse beim Baugewerbe (Tab. 7). Die Revisionen sind in Relation zur Varianz der Zeitreihe beträchtlich; die NSR liegt bei der nominalen Bruttowertschöpfung in sechs, bei der realen sogar bei zehn Ländern bei Eins oder darüber. Zwar ist das Arbeitsvolumen ebenfalls revisionsanfälliger als beispielsweise im Verarbeitenden Gewerbe, sie sind aber auch hier die im Vergleich zur Wertschöpfung die verlässlichere Angabe. Die erste Veröffentlichung der Arbeitsproduktivität kann man getrost als wertlos bezeichnen, nur bei Mecklenburg-Vorpommern ist die NSR kleiner 1, bei elf Ländern übersteigt das Ausmaß der Revision die Varianz der Veränderungsraten im mehr als das Doppelte. 
Tab. 7 Revisionen der VGR der Länder für das Baugewerbe. Veränderungen gegenüber dem Vorjahr; Erste Originärberechnung gegenüber erster Fortschreibung. (Quelle: Eigene Berechnungen nach Angaben des Arbeitskreises VGR der Länder)

\begin{tabular}{|c|c|c|c|c|c|c|c|c|}
\hline & \multicolumn{2}{|c|}{$\begin{array}{l}\text { BWS } \\
\text { nominal }^{\text {a }}\end{array}$} & \multicolumn{2}{|l|}{$\begin{array}{l}\text { BWS } \\
\text { real }^{\mathrm{a}}\end{array}$} & \multicolumn{2}{|c|}{ Arbeitsvolumen $^{\mathrm{b}}$} & \multicolumn{2}{|c|}{ Arbeitsproduktivität ${ }^{C}$} \\
\hline & MAR & NSR & MAR & NSR & MAR & NSR & MAR & NSR \\
\hline Baden-Württemberg & 2,9 & 0,93 & 3,0 & 1,25 & 0,6 & 0,35 & 3,5 & 2,34 \\
\hline Bayern & 3,5 & 1,41 & 3,4 & 1,67 & 0,9 & 0,77 & 4,1 & 2,72 \\
\hline Berlin & 3,7 & 0,43 & 3,8 & 0,52 & 1,2 & 1,06 & 4,5 & 1,81 \\
\hline Brandenburg & 3,0 & 0,28 & 2,8 & 0,36 & 1,1 & 0,45 & 3,0 & 2,01 \\
\hline Bremen & 8,3 & 3,18 & 7,8 & 3,17 & 2,0 & 0,57 & 7,9 & 2,39 \\
\hline Hamburg & 3,9 & 1,27 & 4,1 & 1,72 & 1,3 & 0,35 & 4,3 & 1,59 \\
\hline Hessen & 4,1 & 1,04 & 3,9 & 1,29 & 0,9 & 0,92 & 4,7 & 2,35 \\
\hline $\begin{array}{l}\text { Mecklenburg-Vor- } \\
\text { pommern }\end{array}$ & 5,1 & 0,56 & 4,8 & 0,65 & 1,2 & 0,44 & 5,5 & 0,91 \\
\hline Niedersachsen & 3,2 & 0,86 & 3,1 & 1,06 & 0,4 & 0,09 & 3,3 & 1,99 \\
\hline Nordrhein-Westfalen & 3,0 & 0,68 & 2,8 & 1,06 & 0,9 & 0,58 & 3,6 & 2,44 \\
\hline Rheinland-Pfalz & 3,3 & 0,72 & 3,3 & 1,00 & 1,0 & 0,37 & 3,5 & 3,87 \\
\hline Saarland & 4,9 & 2,45 & 4,6 & 2,31 & 1,3 & 1,20 & 5,0 & 3,01 \\
\hline Sachsen & 4,0 & 0,46 & 3,7 & 0,58 & 0,7 & 0,37 & 3,8 & 6,32 \\
\hline Sachsen-Anhalt & 3,3 & 0,37 & 2,8 & 0,47 & 0,7 & 0,20 & 3,5 & 4,27 \\
\hline Schleswig-Holstein & 4,3 & 1,20 & 4,1 & 1,23 & 1,2 & 0,44 & 3,4 & 2,00 \\
\hline Thüringen & 2,7 & 0,43 & 2,6 & 0,59 & 0,8 & 0,27 & 3,3 & 1,88 \\
\hline Bund & 2,4 & 0,47 & 2,1 & 0,65 & 0,6 & 0,49 & 2,7 & 2,34 \\
\hline
\end{tabular}

a2001 bis 2017

b2006 bis 2017

${ }^{c}$ Reale Bruttowertschöpfung je Erwerbstätigenstunde; 2006 bis 2017

$\mathrm{Zu}$ beachten ist allerdings, dass beim Baugewerbe die zuerst veröffentlichten Angaben auch für den Bund weniger verlässlich sind als die für das Verarbeitende Gewerbe. Dazu trägt wahrscheinlich bei, dass die Bedeutung von Ausbauleistungen gegenüber dem Neubau zugenommen hat und das Ausbaugewerbe aufgrund seiner eher kleinbetrieblichen Struktur in den kurzfristig verfügbaren Statistiken unterrepräsentiert ist. Zudem sind durch die Dienstleistungsfreiheit in der EU viele ausländische Unternehmen auf deutschen Baustellen tätig, die teilweise durch die Maschen der Statistik fallen.

In den Dienstleistungsbereichen schließlich fallen die Revisionen in der Summe weniger ins Gewicht als im Baugewerbe, sie sind aber ausgeprägter als im Verarbeitenden Gewerbe (Tab. 8). Die NSR liegt beim nominalen und beim realen BIP in einer Reihe von Fällen über Eins. Auch hier sind die Revisionen beim Arbeitsvolumen gering, was wiederum kräftige Revisionen der Arbeitsproduktivität impliziert.

Da der Dienstleistungsbereich sehr heterogen ist, auch bezüglich der Datenverfügbarkeit, wäre es aufschlussreich zu erfahren, wie sich die Revisionspraxis zwischen den Dienstleistungssektoren unterscheidet. Aufgrund der Umstellung der Wirtschaftszweigesystematik im Zuge der Generalrevision 2011 ist allerdings ein Vergleich von erster Fortschreibung und erster Originärberechnung nur für sieben Jahre möglich, und dies auch nur für drei Bereiche des Dienstleistungssektors (Tab. 9). In 
Tab. 8 Revisionen der VGR der Länder für die Dienstleistungsbereiche. Veränderungen gegenüber dem Vorjahr; Erste Originärberechnung gegenüber erster Fortschreibung. (Quelle: Eigene Berechnungen nach Angaben des Arbeitskreises VGR der Länder)

\begin{tabular}{lllllllll}
\hline & \multicolumn{2}{l}{$\begin{array}{l}\text { BWS } \\
\text { nominal }\end{array}$} & & \multicolumn{2}{l}{$\begin{array}{l}\text { BWS } \\
\text { real }^{\mathrm{a}}\end{array}$} \\
& MAR & NSR & MAR & NSR & MAR & NSR & MAR & NSR \\
\hline Baden-Württemberg & 1,0 & 0,66 & 1,2 & 0,61 & 0,4 & 0,21 & 0,9 & 0,99 \\
Bayern & 0,7 & 0,59 & 0,7 & 0,52 & 0,4 & 0,30 & 0,9 & 0,80 \\
Berlin & 0,9 & 0,37 & 1,1 & 0,46 & 0,5 & 0,56 & 0,9 & 1,63 \\
Brandenburg & 1,4 & 1,07 & 1,1 & 1,02 & 0,6 & 0,43 & 1,3 & 1,17 \\
Bremen & 1,2 & 0,44 & 1,0 & 0,46 & 0,5 & 0,21 & 1,2 & 0,75 \\
Hamburg & 1,3 & 0,56 & 1,4 & 1,14 & 0,4 & 0,36 & 1,5 & 1,43 \\
Hessen & 1,1 & 0,84 & 1,3 & 0,64 & 0,5 & 0,30 & 1,2 & 0,80 \\
Mecklenburg-Vor- & 1,1 & 1,29 & 1,3 & 1,55 & 0,8 & 0,59 & 1,4 & 1,21 \\
pommern & & & & & & & & Arbeitsproduktivität $^{\mathrm{c}}$ \\
Niedersachsen & 1,0 & 1,22 & 1,2 & 1,06 & 0,4 & 0,24 & 1,2 & 1,14 \\
Nordrhein-Westfalen & 0,8 & 0,46 & 0,9 & 0,56 & 0,3 & 0,14 & 0,9 & 1,41 \\
Rheinland-Pfalz & 1,0 & 0,73 & 0,9 & 0,84 & 0,4 & 0,17 & 0,9 & 1,12 \\
Saarland & 1,0 & 0,93 & 0,9 & 0,46 & 0,5 & 0,27 & 0,8 & 0,66 \\
Sachsen & 0,7 & 0,75 & 0,6 & 0,47 & 0,6 & 0,54 & 0,9 & 1,18 \\
Sachsen-Anhalt & 0,8 & 0,46 & 0,8 & 0,60 & 0,5 & 0,28 & 0,8 & 1,89 \\
Schleswig-Holstein & 0,7 & 0,54 & 0,9 & 0,70 & 0,4 & 0,17 & 0,9 & 0,96 \\
Thüringen & 1,0 & 0,87 & 0,8 & 1,22 & 0,5 & 0,22 & 1,2 & 0,97 \\
Bund & 0,5 & 0,43 & 0,7 & 0,49 & 0,3 & 0,14 & 0,7 & 0,91 \\
\hline
\end{tabular}

a2001 bis 2016

b2005 bis 2016

${ }^{\mathrm{c}}$ Reale Bruttowertschöpfung je Erwerbstätigenstunde; 2005 bis 2016

den Abschnitten G-J der Wirtschaftszweigesystematik findet man in diesem Zeitraum in der Summe - gemessen an MAR - im allgemeinen die höchsten, in den Abschnitten O-T die geringsten Revisionen. Da jedoch in letztgenanntem Bereich die Varianz der Raten gering ist, ist dort die NSR höher. Bei alledem gibt es allerdings Unterschiede zwischen den Ländern.

\section{Folgerungen}

Die vorstehenden Ausführungen zeigen, dass die VGR der Länder erheblichen Revisionen unterliegen. Dies verringert deren Informationsgehalt bezüglich der Einschätzung der wirtschaftlichen Lage der einzelnen Länder; bei einigen Ländern geht der Informationsgehalt der zuerst veröffentlichen Werte für manche Sektoren gegen Null. Zudem zeigt die mangelnde Rangstabilität, dass die zuerst veröffent- 
Tab. 9 Revisionen der der realen Bruttowertschöpfung in Untergruppen des Dienstleistungssektors 2011 bis 2017; Veränderungen gegenüber dem Vorjahr; Erste Originärberechnung gegenüber erster Fortschreibung. (Quelle: Eigene Berechnungen nach Angaben des Arbeitskreises VGR der Länder)

\begin{tabular}{lllllll}
\hline & \multicolumn{2}{l}{ Abschnitte G-J } & \multicolumn{2}{c}{ Anschnitte K-N K $^{\mathrm{b}}$} & \multicolumn{2}{c}{ Abschnitte O-T } \\
& MAR & NSR & MAR & NSR & MAR & NSR \\
\hline Baden-Württemberg & 1,1 & 0,48 & 1,5 & 1,50 & 0,7 & 0,70 \\
Bayern & 0,9 & 0,44 & 1,3 & 0,92 & 0,7 & 0,85 \\
Berlin & 1,2 & 0,68 & 1,3 & 1,02 & 1,6 & 0,94 \\
Brandenburg & 2,6 & 1,40 & 1,8 & 1,53 & 1,0 & 1,03 \\
Bremen & 2,6 & 1,40 & 1,7 & 0,91 & 1,2 & 1,53 \\
Hamburg & 2,1 & 2,28 & 2,3 & 1,73 & 1,4 & 1,93 \\
Hessen & 2,4 & 0,98 & 1,7 & 2,95 & 0,8 & 3,09 \\
Mecklenburg-Vorpom- & 2,7 & 1,28 & 1,8 & 1,59 & 0,7 & 0,89 \\
mern & & & & & & 0,6 \\
Niedersachsen & 1,8 & 1,74 & 1,8 & 0,84 & 0,6 & 0,80 \\
Nordrhein-Westfalen & 1,0 & 0,40 & 1,5 & 1,16 & 1,0 & 1,14 \\
Rheinland-Pfalz & 2,5 & 1,49 & 1,6 & 1,06 & 1,0 & 1,19 \\
Saarland & 1,5 & 0,65 & 1,5 & 0,76 & 0,8 & 1,68 \\
Sachsen & 1,1 & 0,28 & 0,8 & 0,74 & 0,8 & 0,88 \\
Sachsen-Anhalt & 1,8 & 1,85 & 0,9 & 1,11 & 1,2 & 1,13 \\
Schleswig-Holstein & 1,7 & 0,70 & 1,0 & 1,87 & 0,8 & 0,32 \\
Thüringen & 2,9 & 0,67 & 1,4 & 0,75 & 1,5 & 2,41 \\
Bund & 0,8 & 0,44 & 1,1 & 1,40 & 0,5 & 0,51 \\
\hline
\end{tabular}

${ }^{a}$ Handel, Verkehr und Lagerei, Gastgewerbe, Information und Kommunikation

${ }^{\mathrm{b}}$ Finanz-, Versicherungs- und Unternehmensdienstleister sowie Grundstücks- und Wohnungswesen 'Öffentliche und sonstige Dienstleister, Erziehung und Gesundheit, Private Haushalte

lichten Werte keine belastbaren Aussagen zur „tatsächlichen“ Wachstumsposition eines Landes im Spektrum der anderen Länder zulassen. ${ }^{10}$

Dabei zeigt die Revisionsanalyse zwei Ansatzpunkte auf, die VGR der Länder weniger revisionsanfällig und damit aussagekräftiger zu machen. Zum einen zeigt die Analyse, dass die Revisionen beim Arbeitseinsatz (sowohl gemessen an der Zahl der Erwerbstätigen als auch am Arbeitsvolumen) deutlich geringer sind als beim BIP bzw. der Bruttowertschöpfung. Dies ist insofern plausibel, als sich der Arbeitseinsatz, anders als die kapitalbezogene Wertschöpfungskomponenten, bereits aus den kurzfristig verfügbaren Statistiken recht trennscharf nach Ländern abgrenzen lässt. Zum anderen schlägt sich das Zusammenspiel von geringen Revisionen beim Arbeitseinsatz und starken Revisionen bei BIP bzw. Wertschöpfung in äußerst kräftigen Revisionen der Arbeitsproduktivität nieder, und dies insbesondere auch auf der Ebene von Wirtschaftszweigen.

Aus diesen Beobachtungen lassen sich zwei Empfehlungen ableiten.

${ }^{10}$ Erste Vergleiche der Ergebnisse der VGR der Länder mit der Einschätzung der Wirtschaftslage durch die Unternehmen in fünf Ländern lassen vermuten, dass die in den VGR ausgewiesenen Wachstumsunterscheide größer sind als die Unterschiede in der Wahrnehmung der Wirtschaftslage durch die Unternehmen (Döhrn 2019b). 
Erstens sollte bei der Berechnung der VGR der Länder die Arbeitsmarktseite ein größeres Gewicht besitzen. Dies empfiehlt sich auch angesichts der Tatsache, dass die Aufschlüsselung kapitalbezogener Wertschöpfungskomponenten insbesondere in großen Unternehmensverbünden angesichts der zunehmenden Komplexität der inneren Verflechtungen zunehmend schwierig wird. Dies gilt bereits für die Zuordnung der Wirtschaftsleistung multinationaler Unternehmen zu Staaten (Allafi et al. 2017), und dürfte bei der Aufschlüsselung der Kapitaleinkommen deutschlandweit tätiger Unternehmen auf Bundesländer nicht einfacher sein.

Zweitens sollten Veränderungen der Arbeitsproduktivität als Kontrollvariable zur Überprüfung der Plausibilität des Rechenwerks verwendet werden. In der Arbeitsproduktivität ,,prallen“ die Ergebnisse der Erwerbstätigenrechnung der Länder und der VGR der Länder aufeinander. Da es nicht unplausibel ist anzunehmen, dass in einer wettbewerblich organisierten und im internationalen Wettbewerb stehenden Volkswirtschaft alle Unternehmen einer Branche eine im Trend ähnliche Produktivitätsentwicklung aufweisen, sollten Unterschiede zwischen den Bundesländern gut begründbar sein, z. B. durch Unterschiede in der Kapazitätsauslastung oder in Unternehmenscharakteristiken. Mit Skepsis sollte man insbesondere großen Sprüngen in der Produktivität von Jahr zu Jahr begegnen.

Danksagung Der Verfasser dankt dem Arbeitskreis Volkswirtschaftliche Gesamtrechnungen der Länder für die Bereitstellung der Echtzeit-Daten der VGR der Länder. Der Verfasser dankt Stefan Boes, Philip Reimers, Dirk Schlotböller und einem anonymen Gutachter für ihre Anmerkungen zu früheren Fassungen dieses Beitrags.

Funding Open Access funding enabled and organized by Projekt DEAL.

Open Access Dieser Artikel wird unter der Creative Commons Namensnennung 4.0 International Lizenz veröffentlicht, welche die Nutzung, Vervielfältigung, Bearbeitung, Verbreitung und Wiedergabe in jeglichem Medium und Format erlaubt, sofern Sie den/die ursprünglichen Autor(en) und die Quelle ordnungsgemäß nennen, einen Link zur Creative Commons Lizenz beifügen und angeben, ob Änderungen vorgenommen wurden.

Die in diesem Artikel enthaltenen Bilder und sonstiges Drittmaterial unterliegen ebenfalls der genannten Creative Commons Lizenz, sofern sich aus der Abbildungslegende nichts anderes ergibt. Sofern das betreffende Material nicht unter der genannten Creative Commons Lizenz steht und die betreffende Handlung nicht nach gesetzlichen Vorschriften erlaubt ist, ist für die oben aufgeführten Weiterverwendungen des Materials die Einwilligung des jeweiligen Rechteinhabers einzuholen.

Weitere Details zur Lizenz entnehmen Sie bitte der Lizenzinformation auf http://creativecommons.org/ licenses/by/4.0/deed.de.

\section{Literatur}

AK VGRdL - Arbeitskreis Volkswirtschaftliche Gesamtrechnungen der Länder (2017) Methodenbeschreibung ESVG 2010 / Revision 2014

AK VGRdL - Arbeitskreis Volkswirtschaftliche Gesamtrechnungen der Länder (2020) Qualitätsbericht regionale Volkswirtschaftliche Gesamtrechnungen. AK VGRdL, Stuttgart

Allafi S, Jung S, Spies V (2017) Globalisierung in der amtlichen Statistik. Wirtsch Stat 2017(55):130-148

Döhrn R (2019a) Revisionen der Volkswirtschaftlichen Gesamtrechnungen und ihre Auswirkungen auf Prognosen. Asta Wirtsch Sozialstat Arch. https://doi.org/10.1007/s11943-019-00251-x

Döhrn R (2019b) Zur Aussagekraft der VGR der Länder - Revisionspraxis und Verbesserungsvorschläge. RWI-Materialien 132. RWI, Essen 
LSN - Landesamt für Statistik Niedersachsen (2018) Bruttoinlandsprodukt in Niedersachsen um 2,5\% gestiegen (Pressemitteilung 26/18 vom 28. März 2018)

Statistisches Bundesamt (2016) Volkswirtschaftliche Gesamtrechnungen. Inlandsprodukt und Nationaleinkommen nach ESVG 2010. Methoden und Grundlagen. Fachserie 18, Reihe. DeStatis, Wiesbaden, S 30

Hinweis des Verlags Der Verlag bleibt in Hinblick auf geografische Zuordnungen und Gebietsbezeichnungen in veröffentlichten Karten und Institutsadressen neutral. 